\title{
Lanczos-based equation-of-motion coupled-cluster singles-and-doubles approach to the total photoionization cross section of valence excited states
}

Cabral Tenorio, Bruno Nunes; Chaer Nascimento, Marco Antonio; Rocha, Alexandre Braga; Coriani, Sonia

Published in:

Journal of Chemical Physics

Link to article, DOI:

$10.1063 / 1.5125125$

Publication date:

2019

Document Version

Publisher's PDF, also known as Version of record

Link back to DTU Orbit

Citation $(A P A)$ :

Cabral Tenorio, B. N., Chaer Nascimento, M. A., Rocha, A. B., \& Coriani, S. (2019). Lanczos-based equation-ofmotion coupled-cluster singles-and-doubles approach to the total photoionization cross section of valence excited states. Journal of Chemical Physics, 151(18), [184106]. https://doi.org/10.1063/1.5125125

\section{General rights}

Copyright and moral rights for the publications made accessible in the public portal are retained by the authors and/or other copyright owners and it is a condition of accessing publications that users recognise and abide by the legal requirements associated with these rights.

- Users may download and print one copy of any publication from the public portal for the purpose of private study or research.

- You may not further distribute the material or use it for any profit-making activity or commercial gain

- You may freely distribute the URL identifying the publication in the public portal 


\section{Lanczos-based equation-of-motion coupled-cluster singles-and-doubles approach to the total photoionization cross section of valence excited states}

Cite as: J. Chem. Phys. 151, 184106 (2019); https://doi.org/10.1063/1.5125125

Submitted: 20 August 2019 . Accepted: 29 October 2019 . Published Online: 12 November 2019

Bruno Nunes Cabral Tenorio (D), Marco Antonio Chaer Nascimento, Alexandre Braga Rocha (D), and Sonia Coriani (iD)
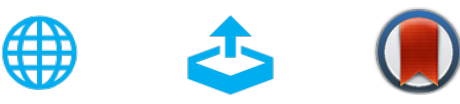

View Online

Export Citation

\section{ARTICLES YOU MAY BE INTERESTED IN}

Rank reduced coupled cluster theory. II. Equation-of-motion coupled-cluster singles and doubles

The Journal of Chemical Physics 151, 164121 (2019); https://doi.org/10.1063/1.5121867

The simulation of X-ray absorption spectra from ground and excited electronic states using core-valence separated DFT/MRCI

The Journal of Chemical Physics 151, 144104 (2019); https://doi.org/10.1063/1.5110418

Adventures in DFT by a wavefunction theorist

The Journal of Chemical Physics 151, 160901 (2019); https://doi.org/10.1063/1.5116338

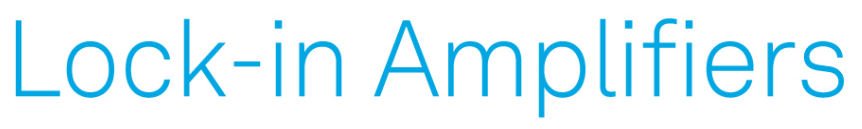

... and more, from DC to $600 \mathrm{MHz}$ Watch 


\title{
Lanczos-based equation-of-motion coupled-cluster singles-and-doubles approach to the total photoionization cross section of valence excited states
}

\author{
Cite as: J. Chem. Phys. 151, 184106 (2019); doi: 10.1063/1.5125125 \\ Submitted: 20 August 2019 - Accepted: 29 October 2019 • \\ Published Online: 12 November 2019
}

\begin{abstract}
Bruno Nunes Cabral Tenorio,,a) (D) Marco Antonio Chaer Nascimento, Alexandre Braga Rocha, ${ }^{\text {(D) }}$ and Sonia Coriani ${ }^{2, b)}$ (D)
\end{abstract}

\author{
AFFILIATIONS \\ ${ }^{1}$ Universidade Federal do Rio de Janeiro, UFRJ, Instituto de Química, Av. Athos da Silveira Ramos, 149, \\ Rio de Janeiro, RJ 21941-909, Brazil \\ ${ }^{2}$ DTU Chemistry, Technical University of Denmark, Kemitorvet, Building 207, DK-2800 Kongens Lyngby, Denmark
}

\author{
a) Electronic mail: b.nunes.bruno@gmail.com \\ b) Electronic mail: soco@kemi.dtu.dk
}

\begin{abstract}
Excitation energies and oscillator strengths of the first two electronically excited states of helium, water, sulfur dioxide, molecular nitrogen, and carbon monoxide were obtained from an asymmetric-Lanczos-based formulation of the equation-of-motion coupled cluster singles and doubles approach. The total photoionization cross sections were generated by two different methodologies: an analytic continuation procedure based on the Padé approximants and the Stieltjes imaging technique. The results are compared with theoretical photoionization cross sections from algebraic diagrammatic construction [ADC(2)] and ADC(2)-x calculations [M. Ruberti et al., J. Chem. Phys. 140, 184107 (2014)] and with available experimental data.
\end{abstract}

Published under license by AIP Publishing. https://doi.org/10.1063/1.5125125

\section{INTRODUCTION}

In recent years, we have witnessed a steady increase in the number of experimental and theoretical works focusing on the light absorption of molecules in electronically excited states. With the advent of attosecond light pulses, photons lying in the ultraviolet (UV) and X-ray spectral regions can now be employed as the probing light in pump-probe spectroscopy experiments reaching the attosecond time scale. ${ }^{1-12}$ The development of new experimental techniques to access information on transient states of atoms and molecules asks for further improvements of the theoretical methods necessary to help in the interpretation of such experiments. The photoionization cross section of transient states may supply valuable information about the electronic and nuclear structure of the molecules. The lack of the continuum asymptotic information in the electronic wave functions computed with full quadratically integrable basis sets (also known as $L^{2}$ basis sets) does not allow for the calculation of phase-shifts necessary to obtain partial photoionization cross sections and angular distributions. Nonetheless, accurate calculations of total photoionization cross sections of molecules in electronically excited states may be of value to guide experimentalists in the choice of suitable candidate transient states of molecules and in the development of new experiments.

Many computational studies of the photoionization/ photoabsorption cross sections of valence and/or core electrons of atoms and molecules in their electronic ground state relying on $L^{2}$ basis sets have been reported employing either the well-known Stieltjes imaging procedure ${ }^{13-18}$ or an analytical continued procedure based on the Padé approximants ${ }^{19-22}$ to describe the continuum part of the spectra. 
As for systems in an electronic excited state, in the 1970s, Yeager, Nascimento, and McKoy ${ }^{23}$ had already obtained the photoionization cross section of the ${ }^{3} \mathrm{~S}$ and ${ }^{1} \mathrm{~S}$ metastable states of helium (as well as the decay rate for the ${ }^{1} S$ metastable state) within the random phase approximation (RPA) by using an analytical continuation procedure based on the Padé approximants. In the past few years, Averbukh and co-workers extended the algebraic diagrammatic construction (ADC)-Stieltjes-Lanczos method to the photoionization of transient valence-excited states ${ }^{7}$ and core-excited states. ${ }^{8}$ Recently, Tenorio et al. ${ }^{24}$ reported the first extension and benchmark of Lanczos-based core-valence-separated coupled cluster approaches exploiting both analytic continuation with Padé approximants and Stieltjes imaging as strategies to compute the inner-shell photoionization cross sections.

Herein, we derive a Lanczos-based approach, at the equationof-motion (EOM) coupled cluster singles and doubles (CCSD) level of theory, ${ }^{25-28}$ to compute the pseudospectra of valence excited states. The pseudospectra thus obtained are subsequently used with both the Stieltjes imaging and the Padé analytic continuation procedures to reconstruct the photoionization cross section of transient states of the water, carbon monoxide, nitrogen, and sulfur dioxide molecules and helium atoms. The results are compared with the theoretical results of Ruberti et al. ${ }^{7}$ at the ADC level and with the available experimental data.

\section{THEORY}

\section{A. Lanczos EOM-CCSD transition strengths between excited states}

Following the notation introduced in Refs. 25, 28, and 29, we write the EOM-CC transition moments, $T_{i j}^{X X}$, between an initial excited state $|i\rangle$ and a final state $|j\rangle$ for an operator (component) $X$ as

$$
T_{i j}^{X X}=\left(L_{i} \mathbf{A}^{X} R_{j}\right)-\left(L_{i} \cdot \xi^{X}\right)\left(\bar{t} \cdot R_{j}\right)-\left(L_{i} \cdot R_{j}\right)\left(\bar{t} \cdot \xi^{X}\right),
$$

where $\bar{t}$ are the ground state Lagrangian multipliers and $\xi^{X}$ is the CC property gradient vector for operator $X{ }^{30}$ By adopting the EOM-CC expression for the transition moments, the computation of transition strengths within the asymmetric Lanczos reformulation of CC response theory (vide infra) is facilitated, since all terms involving the $\mathbf{B}$ matrix transformation-that is, either the response amplitudes $t^{X}\left(\omega_{i}-\omega_{j}\right)$ or the Lagrangian vectors $\bar{N}^{i j}\left(\omega_{i}-\omega_{j}\right)^{30}$-are avoided.

Given the transition moments in Eq. (1), the transition strength $S_{i j}^{X X}$ and the oscillator strength $f_{i j}$ are then written as often done in CC response theory ${ }^{30,31}$

$$
\begin{aligned}
S_{i j}^{X X} & =T_{i j}^{X X} \cdot T_{j i}^{X X}, \\
f_{i j} & =\frac{2}{3}\left(\omega_{j}-\omega_{i}\right)\left(S_{i j}^{X X}+S_{i j}^{Y Y}+S_{i j}^{Z Z}\right) .
\end{aligned}
$$

Within the EOM-CC framework for pure CC models, the right and left excitation vectors and their excitation energies $\left(\omega_{i}, \omega_{j}\right)$ are the same of CC linear response, ${ }^{30,31}$ obtained as eigenvectors and eigenvalues of the CC Jacobian matrix A,

$$
L_{j} \mathbf{A}=\omega_{f} L_{j}, \quad \mathbf{A} R_{j}=\omega_{j} R_{j},
$$

under the biorthogonality condition $L_{j} R_{l}=\delta_{j l}$. The Jacobian matrix A is defined as

$$
A_{\mu v}=\left\langle\mu\left|\exp (-T)\left[H, \tau_{v}\right] \exp (T)\right| \mathrm{HF}\right\rangle \equiv\left\langle\mu\left|\left[H^{T}, \tau_{v}\right]\right| \mathrm{HF}\right\rangle,
$$

where we also introduced the similarity transformed Hamilton operator, $H^{T}=\exp (-T) X \exp (T)$. The cluster operator $T$ is, as usual, a sum of excitation operators, $\tau_{\mu}$, each one weighted by its corresponding amplitude $t_{\mu}, T=\sum_{\mu} t_{\mu} \tau_{\mu}$. The excited Slater determinant $|\mu\rangle$ is generated by the excitation operator $\tau_{\mu}$ out of the reference Hartree-Fock state, $|\mu\rangle=\tau_{\mu}|\mathrm{HF}\rangle$.

The EOM-CC property Jacobian matrix $\mathbf{A}^{X}$ in Eq. (1) is different from the one of CC response theory (below identified with superscript RSP), but it can be easily obtained from it with minor modifications, ${ }^{29}$

$$
\mathbf{A}_{\mu \nu}^{X}=\mathbf{A}_{\mu v}^{X, \mathrm{RSP}}+\left\langle\mu\left|\tau_{v} X^{T}\right| \operatorname{HF}\right\rangle\left(1-\delta_{\mu v}\right),
$$

where the elements $\mathbf{A}_{\mu \nu}^{X, \text { RSP }}$ are defined as in Eq. (5), with the operator $X$ in place of the Hamiltonian $H$ (per definition, and $\mathbf{A}^{X, \mathrm{RSP}}$ is the derivative of the CC Jacobian with respect to the strength of the perturbation $X$ ). Equation (6) also contains the similarity-transformed operator $X^{T}=\exp (-T) X \exp (T)$.

Coriani and co-workers ${ }^{32,33}$ have proposed to use an asymmetric Lanczos algorithm to solve Eq. (4), yielding a discretized spectrum covering the whole frequency range. In the algorithm, a truncated tridiagonal representation $\mathbf{T}$ of the Jacobian matrix $\mathbf{A}$ is built and then straightforwardly diagonalized. The leading size of $\mathbf{T}$ is the chain length $k \ll n$, where $n$ is the full dimension of the excitation space. The nonzero elements of the tridiagonal matrix $\mathbf{T}=\mathbf{P}^{T} \mathbf{A Q}$, where $\mathbf{P}^{T} \mathbf{Q}=\mathbf{1}$, are given by

$$
\begin{gathered}
T_{l l} \equiv \alpha_{l}=\mathbf{p}_{l}^{T} \mathbf{A} \mathbf{q}_{l}, \quad T_{l+1, l} \equiv \beta_{l}=\sqrt{\mathbf{p}_{l+l}^{T} \mathbf{q}_{l+1}}, \\
T_{l, l+1} \equiv \gamma_{l}=\operatorname{sgn}\left\{\mathbf{p}_{l+1}^{T} \mathbf{q}_{l+1}\right\} \beta_{l}
\end{gathered}
$$

with

$$
\begin{gathered}
\mathbf{q}_{l+1}=\beta_{l}^{-1}\left(\mathbf{A q}_{l}-\gamma_{l-1} \mathbf{q}_{l-1}-\alpha_{l} \mathbf{q}_{l}\right), \\
\mathbf{p}_{l+1}^{T}=\gamma_{l}^{-1}\left(\mathbf{p}_{l}^{T} \mathbf{A}-\beta_{l-1} \mathbf{p}_{l-1}^{T}-\alpha_{l} \mathbf{p}_{l}^{T}\right),
\end{gathered}
$$

where $\mathbf{p}_{i}$ and $\mathbf{q}_{i}$ are columns of the (rectangular, $k \times n$ ) matrices $\mathbf{P}$ and $\mathbf{Q}$, respectively. The initial seeds $\mathbf{q}_{1}$ and $\mathbf{p}_{1}$ were chosen as (bi)normalized CC vectors $\xi^{X}$ and $\eta^{X}$, giving direct access to the dipole-allowed transitions, i.e., an effective excitation spectrum. The effective excitation spectrum generated by the diagonalization of $\mathbf{T}$ is known to converge from the bottom and from the top toward the exact excitation spectrum. ${ }^{32-34}$

Total photoionization cross sections have been extensively studied applying both Stieltjes imaging and analytic continuation procedures to the effective ground state excitation pseudospectrum yielded by the asymmetric Lanczos algorithm and with oscillator strengths formulated according to CC response theory. ${ }^{15,16,21,24}$

Herein, we are interested in obtaining the total photoionization cross sections for a valence excited state. Specifically, we aim at generating the pseudospectrum from a chosen excited state employing an asymmetric Lanczos algorithm and with strengths corresponding to the EOM-CCSD ones given in Eq. (1).

Our procedure for calculating the excited-state pseudospectrum within the EOM-CCSD framework, which bears similarities 
to the one adopted by Ruberti et al. in the ADC context, can be summarized as follows:

1. First, we calculate the initial excitation vectors $\left(R_{i}\right.$ and $\left.L_{i}\right)$ via a regular Davidson diagonalization of Eq. (4).

2. Next, we select the Lanczos seeds and build the tridiagonal representation of the CC Jacobian $\mathbf{T}$ so as to span the space of final excited states $(j)$ that are dipole allowed when starting from the initial.

3. We diagonalize the tridiagonal matrix $\mathbf{T}$, conveniently truncated to a dimension (chain length) $k$, to obtain the final space pseudospectrum.

4. We compute the oscillator strengths between the initial state, state $i$, and the final states $j$ from the Lanczos eigenvectors and eigenvalues of states $j$, and the Davidson eigenvectors and eigenvalue of state $i$.

Crucial steps in our procedure are the proper choice of initial Lanczos seeds in order to generate the pseudospectrum in the final symmetry space [step (2)] and the computation of the EOM-CCSD transition strengths between excited states [step (4)].

As Lanczos starting vectors, we use the following normalized $\mathbf{q}_{1}$ and $\mathbf{p}_{1}^{T}$ vectors:

$$
\begin{aligned}
& \mathbf{q}_{1}=\mathbf{Q} \mathbf{e}_{1}=x_{q}^{-1}\left[\mathbf{A}^{X} R_{i}-\left(\bar{t} \cdot R_{i}\right) \xi^{X}\right], \\
& \mathbf{p}_{1}^{T}=\mathbf{e}_{1}^{T} \mathbf{P}^{T}=x_{p}^{-1}\left[L_{i} \mathbf{A}^{X}-\left(L_{i} \cdot \xi^{X}\right) \bar{t}\right],
\end{aligned}
$$

where $x_{q}$ and $x_{p}$ are the normalization factors and $\mathbf{e}_{m}$ a vector of length $k$ whose $m$ th element is equal to 1 and all other elements are equal to zero. Note that these seeds depend on the choice of initial valence excited state $i$.

The EOM-CCSD right-transformed vector $\left(\mathrm{A}^{X} R_{i}\right)$ is obtained as described in the Appendix. The seeds in Eq. (9) were defined based on Eq. (1), where the last term was neglected since it eventually will not contribute because of the biorthogonality condition between excited states. With this choice of Lanczos seeds, we can straightforwardly calculate the EOM-CCSD transition strength between two excited states in Eq. (1) as,

$$
\begin{aligned}
T_{i j}^{X X} \cdot T_{j i}^{X X}=\left({ }^{i} x_{q} L_{j} \mathbf{q}_{1}\right) \cdot\left({ }^{i} x_{p} \mathbf{p}_{1}^{T} R_{j}\right) & =\left({ }^{i} x_{q} L_{j}^{(k)} \mathbf{P}^{T} \mathbf{Q} \mathbf{e}_{1}\right)\left({ }^{i} x_{p} \mathbf{e}_{1}^{T} \mathbf{P}^{T} \mathbf{Q} R_{j}^{(k)}\right), \\
S_{i j}^{X X} & ={ }^{i} x_{p}{ }^{i} x_{q} R_{j, 1}^{(k)} L_{j, 1}^{(k)},
\end{aligned}
$$

where we used the orthogonality condition $\mathbf{P}^{T} \mathbf{Q}=\mathbf{1}$ (of dimension $k \times k$ ) and the back-transformations of the Lanczos eigenvectors $L_{j}^{(k)}$ and $R_{j}^{(k)}$ into full space, $R_{j}=\mathbf{Q} R_{j}^{(k)}$ and $L_{j}=L_{j}^{(k)} \mathbf{P}^{T}$. The superscript $i$ in the normalization factors highlights the implicitly built dependence on the initial excited state.

It is worth mentioning that, as in the case of the ground state, ${ }^{32,33}$ the final expression for the transition moment between the excited states shown in Eq. (10) only involves the first element of the Lanczos left and right excitation vectors of the final state, $\mathbf{e}_{1}^{T} R_{j}^{(k)}=R_{j, 1}^{(k)}$ and $L_{j}^{(k)} \mathbf{e}_{1}=L_{j, 1}^{(k)}$, making the computation of the transition moments very simple.

\section{B. Analytic continued calculation of the photoionization cross section of excited states}

Yeager, Nascimento, and McKoy ${ }^{23}$ obtained the photoionization cross sections of the $2{ }^{3} \mathrm{~S}$ and $2{ }^{1} \mathrm{~S}$ metastable states of the helium atom using an analytical continuation procedure based on the Padé approximants. By defining an initial excited state $\left|\lambda_{i}\right\rangle$ from which all one-photon excitation energies $\omega_{i j}=\left(\omega_{j}-\omega_{i}\right)$ and oscillator strengths $f_{i j}$ are generated, $\left\{\omega_{i j}, f_{i j}\right\}_{i<j}$, the photoionization cross section $\sigma_{\lambda_{i}}(\omega)$ is obtained from the imaginary part of the averaged dynamic dipole polarizability function of the excited electronic state,

$$
\sigma_{\lambda_{i}}(\omega)=\frac{4 \pi \omega}{c} \lim _{\eta \rightarrow 0} \operatorname{Im}\left[\bar{\alpha}_{\lambda_{i}}(\omega+i \eta)\right] .
$$

The averaged dynamic dipole polarizability function $\bar{\alpha}_{\lambda_{i}}(z)$ of the excited state $i$ is approximated by a finite sum over the electronic pseudospectrum $\left\{\omega_{i j}, f_{i j}\right\}_{i<j}$ obtained in the $L^{2}$ basis set calculation

$$
\alpha_{\lambda_{i}}(z)=\sum_{j=1}^{k} \frac{f_{i j}}{\omega_{i j}^{2}+z^{2}},
$$

and $z$ is the complex valued frequency.

Here, we build an approximation of Eq. (12) from the set of Lanczos EOM-CCSD excited-to-excited transition energies and oscillator strengths. Then, in the spirit of Ref. 23, having such an approximation in hand, we calculate $\alpha_{\lambda_{i}}(z)$ at a number of points in the complex plane. These points are subsequently fitted by a continued fraction function-which is mathematically equivalent to the Padé approximants-providing a representation of $\alpha_{\lambda_{i}}(z)$ in the complex plane. The imaginary part of $\alpha_{\lambda_{i}}(z)$ on the real axis provides the cross section by Eq. (11).

The set of points in the complex plane is known to be arbitrary and not unique, and here, we are using the recipe previously described in Ref. 20 to select the complex points.

\section{The photoionization cross section of an excited state from Stieltjes imaging}

Another approach to obtain the photoionization cross section from a discrete pseudospectrum obtained with an $\mathrm{L}^{2}$ basis set calculation is the Stieltjes imaging procedure, suggested by Langhoff. ${ }^{35,36}$ Similar to the Padé analytic continuation procedure, it is assumed that the moments $s(k)$ of the continuum oscillator strength

$$
s(k)=\sum_{l}^{\text {discr }} \omega_{l}^{k} f_{l}+\int_{\omega_{T}}^{\infty} \omega^{k} f(\omega) d \omega
$$

can be approximated by the sum of $N$ discrete pseudostates of the continuum, $S(k) \approx \sum_{i}^{N} \bar{\omega}_{i}^{k} \bar{f}_{i}$.

In the Stieltjes imaging, the "primitive" set of $M$ excitation energies and oscillator strengths are transformed in order to obtain a smoothed representation of the continuum spectrum. One first computes a number $(2 r)$ of "spectral moments" $S(-k)$ of the primitive spectrum

$$
S(-k)=\sum_{l=1}^{M} f_{l} \omega_{l}^{-k} ; \quad k=0, \ldots, 2 r-1 .
$$

Based on the results of previous works, ${ }^{15,16,24}$ here,we used $r$ ranging from 2 to 20 and only to plot in the figures the points that are well converged.

From the $2 r$ spectral moments, the discretized spectra of order $m=2, \ldots, r$, called principal pseudospectra, are generated as 
generalized (Gaussian) quadrature points (abscissae) $\omega_{l}^{(m)}$ and weights $f_{l}^{(m)}$,

$$
S(-k)=\sum_{l=1}^{m} f_{l}^{(m)}\left(\omega_{l}^{(m)}\right)^{-k}, \quad k=0, \ldots, 2 m-1 .
$$

From the Gaussian quadrature points and weights, one reconstructs the photoionization cross section spectra numerically as the Stieltjes derivative of the general discretized principal pseudospectra (see Refs. 15, 24, and 37 for a more detailed description of the Stieltjes imaging procedure). Here, we used the computed values $\omega_{i j}$ and $f_{i j}$ as the primitive set in the Stieltjes procedure to get the spectra for cross section points for the initial excited state.

\section{COMPUTATIONAL DETAILS}

The asymmetric Lanczos procedure discussed in Sec. II A for calculating the spectral moments between excited states at the EOMCCSD level has been implemented in a development version of the Dalton program package.

Chain-length subspaces of $k=500$ were used for all molecules considered, adopting Dunning's correlation consistent basis set augcc-pVTZ. ${ }^{39}$ The basis set was enriched with continuum-like basis functions proposed by Kaufmann, Baumeister, and Jungen. ${ }^{40}$ The continuum-like functions were added to the center of mass of each molecule, which included $s, p$, and $d$ functions generated using quantum numbers $n$ ranging from 2 to 8 , as employed in Ref. 24 . The same notation introduced there will be used to indicate such a set, namely, $(7 \mathrm{~s} 7 \mathrm{p} 7 \mathrm{~d})_{n=2-8}$.

The geometries for the water, nitrogen, and carbon monoxide molecules are the same ones used in Ref. 7 , in order to establish a ground for comparison between our results and their ADC ones. Similar to Ref. 7, the water molecule ground state equilibrium geometry and the lowest-energy geometries for the $1{ }^{1} \mathrm{~B}_{1}$ and $1{ }^{1} \mathrm{~A}_{2}$ states were obtained from Ref. 41 calculated with the d-aug-cc-pVQZ basis set at the CASPT2 level. The carbon monoxide experimental ground state equilibrium geometry and the experimental lowest ${ }^{1} \Sigma^{-}$and ${ }^{1} \Pi$ excited states equilibrium geometries were obtained from Ref. 42. For molecular nitrogen, the geometries used here and in Ref. 7 are experimental geometries.

The calculations for the Helium atom have been performed with the Lanczos chain dimension equal to the full excitation space dimension [exact Full Configuration Interaction (FCI) results], using the aug-cc-pVQZ basis complemented with Kaufmann's $(7 \mathrm{~s} 7 \mathrm{p} 7 \mathrm{~d})_{n=2-8}$ continuum-like set.

It is worth mentioning that the photoionization cross section results presented herein have been plotted using the "photon energy," as the energy scale, i.e., the energy of the final excited state minus the energy of the considered transient excitation. This was done in order to consider the photoabsorption by the system already prepared in the transient excited state.

\section{RESULTS}

Table I collects the calculated vertical excitation energies of the initial valence excited states of each system considered in the present study. Corresponding experimental values are also included.
TABLE I. Calculated vertical excitation energies $\omega_{i}$ (in eV) for the initial excited states considered in this work and corresponding experimental vertical excitation energies.

\begin{tabular}{lcc}
\hline \hline Excited state & Vertical $\omega_{i}(\mathrm{eV})$ & Experimental value $(\mathrm{eV})$ \\
\hline $\mathrm{He}^{1} \mathrm{~S}$ & 20.61 & $20.61^{43}$ \\
$\mathrm{He}^{1} \mathrm{P}$ & 21.21 & $21.22^{44}$ \\
$\mathrm{H}_{2} \mathrm{O}^{1} \mathrm{~B}_{1}$ & 7.61 & $7.40^{47}$ \\
$\mathrm{H}_{2} \mathrm{O}^{1} \mathrm{~A}_{2}$ & 9.36 & $9.09^{47}$ \\
$\mathrm{CO}^{1} \Pi$ & 8.62 & $8.51^{48}$ \\
$\mathrm{CO}^{1} \Sigma^{-}$ & 10.08 & $9.88^{48}$ \\
$\mathrm{~N}_{2}{ }^{1} \Pi_{g}$ & 9.44 & $9.31^{50}$ \\
$\mathrm{~N}_{2}{ }^{1} \Sigma_{u}^{-}$ & 10.04 & $9.92^{50}$ \\
$\mathrm{SO}_{2}{ }^{1} \mathrm{~B}_{1}$ & 4.40 & $3.6-5.1^{53}$ \\
$\mathrm{SO}_{2}{ }^{1} \mathrm{~B}_{2}$ & 6.51 & $5.4-7.3^{53}$ \\
\hline \hline
\end{tabular}

\section{A. Helium}

For the two-electron system helium, the CCSD approximation is equivalent to a full $\mathrm{CI}$ (FCI) calculation and the EOM-CCSD results can then be considered as a benchmark. Moreover, there are no vibrational nor geometrical effects, making helium a perfect choice to validate our computational method.

In Fig. 1, we present the computed photoionization cross sections from the (1s2s) ${ }^{1} \mathrm{~S}$ and the $(1 \mathrm{~s} 2 \mathrm{p}){ }^{1} \mathrm{P}$ excited states of the helium atom together with the available experimental values. Experimental photoionization cross sections from the same excited states have been reported in Refs. 43 and 44, respectively, and they are also plotted in Fig. 1.

The calculated excitation energies from the ground state to the ${ }^{1} \mathrm{~S}$ and the ${ }^{1} \mathrm{P}$ excited states are $20.61 \mathrm{eV}$ and $21.21 \mathrm{eV}$, respectively, in excellent agreement with the experimental values of $20.61 \mathrm{eV}^{43}$ and $21.22 \mathrm{eV}^{44}$ (see Table I). The accuracy of the calculated excitation energies reflects the above-mentioned equivalence between FCI and CCSD in this case.

The calculated photoionization cross section from the ${ }^{1} \mathrm{~S}$ state (the top panel of Fig. 1) is in excellent agreement with the experimental points. The same agreement can be observed for the cross section from the ${ }^{1} \mathrm{P}$ state (the bottom panel of Fig. 1). For both states, there is a reasonable agreement between the analytic Padé curve and the Stieltjes points, but the Stieltjes points for the ${ }^{1} \mathrm{P}$ excited state are more scattered, possibly an indication of poor convergence of the principal pseudospectrum in this case. Our results are also in good agreement with other calculations by Yeager et al. ${ }^{23}$ and Jacobs.

\section{B. Water}

Our EOM-CCSD results for water are compared with the ones obtained by Ruberti et al. ${ }^{7}$ at the $\mathrm{ADC}(2)$ and $\mathrm{ADC}(2)$-x levels in Fig. 2.

Ruberti et al. ${ }^{7}$ also reported results at the ADC(1) level, which proved to be inadequate to obtain the photoionization cross sections, even at the qualitative level, if compared to the more accurate $\operatorname{ADC}(2)$ and $\operatorname{ADC}(2)-x$ levels. These results ${ }^{7}$ showed the need to include double excitation effects in order to obtain reasonable 

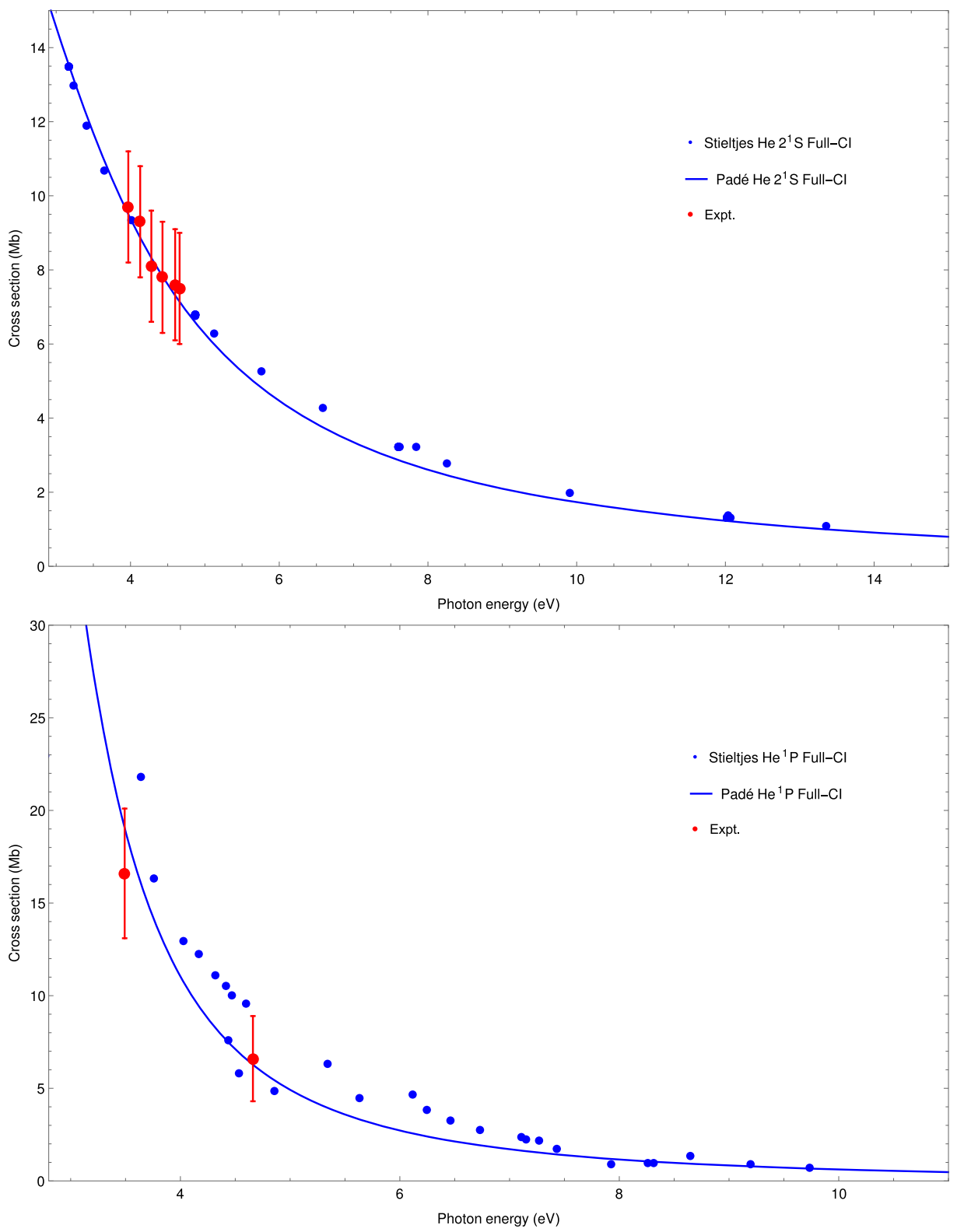

FIG. 1. He. $\mathrm{FCl}$ photoionization cross sections from the $1 \mathrm{~s} 2 \mathrm{~s}^{1} \mathrm{~S}$ (top) and $1 \mathrm{~s} 2 \mathrm{p}$ ${ }^{1} \mathrm{P}$ (bottom) excited states, obtained via the Padé and Stieltjes imaging procedures. The experimental points were taken from Refs. 43 and 44 .

photoionization cross sections of transient states. In addition, the authors have found that the inclusion of coupling between the double excitations in $\mathrm{ADC}(2)$ theory, leading to the $\mathrm{ADC}(2)-\mathrm{x}$ approach, makes a substantial difference in the final results.

Our EOM-CCSD photoionization cross sections from the two lowest excited states of water, ${ }^{1} B_{1}$ and ${ }^{1} A_{2}$, are shown in Figs. 2 and 3 , respectively, together with the $\mathrm{ADC}(2)$ and $\mathrm{ADC}(2)$-x results by Ruberti et al. ${ }^{7}$ in order to have some ground for comparison. To compare our results with the $\operatorname{ADC}(2)$ and $\operatorname{ADC}(2)-x$ ones, we used the same geometries as those reported by Ruberti et al. ${ }^{7}$ The results obtained at the ground state equilibrium geometry are shown in the top panels of Figs. 2 and 3, and the ones obtained at the lowest-energy geometries of the excited states, as calculated in Ref. 41 , are shown in the bottom panel of the same figures.

The vertical excitation energy at the ground state geometry for the ${ }^{1} B_{1}$ state was calculated at $7.61 \mathrm{eV}$, while a previous EOM-CCSD ${ }^{46}$ study, employing an aug-cc-pVTZ basis set, reported $7.58 \mathrm{eV}$. For the ${ }^{1} A_{2}$ state, we calculated the excitation energy at $9.36 \mathrm{eV}$, whereas the previous EOM-CCSD result ${ }^{46}$ is $9.35 \mathrm{eV}$. Our vertical excitation energies are in good agreement with experimental values (7.40 eV and $9.09 \mathrm{eV}^{47}$ for the ${ }^{1} B_{1}$ and ${ }^{1} A_{2}$ excited states, respectively; see Table I). Compared to the $\operatorname{ADC}(2)$ and $\operatorname{ADC}(2)-x$ results ${ }^{7}$ (obtained with a different basis set), the differences are of the order of 5\%. The adiabatic excitation energies at the lowest-energy 


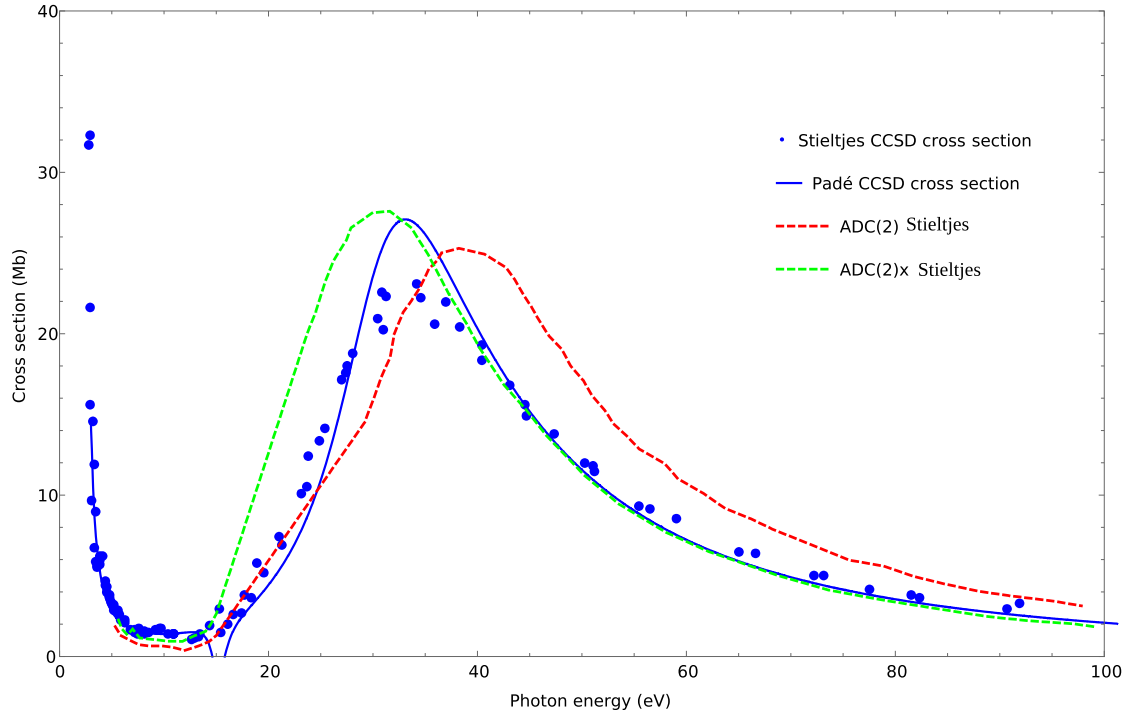

FIG. 2. $\mathrm{H}_{2} \mathrm{O}$. EOM-CCSD total photoionization cross sections from the ${ }^{1} B_{1}$ excited state at the ground state equilibrium geometry (top) and at the ${ }^{1} B_{1}$ lowest-energy geometry of the excitedstate of Ref. 41 (bottom), obtained by

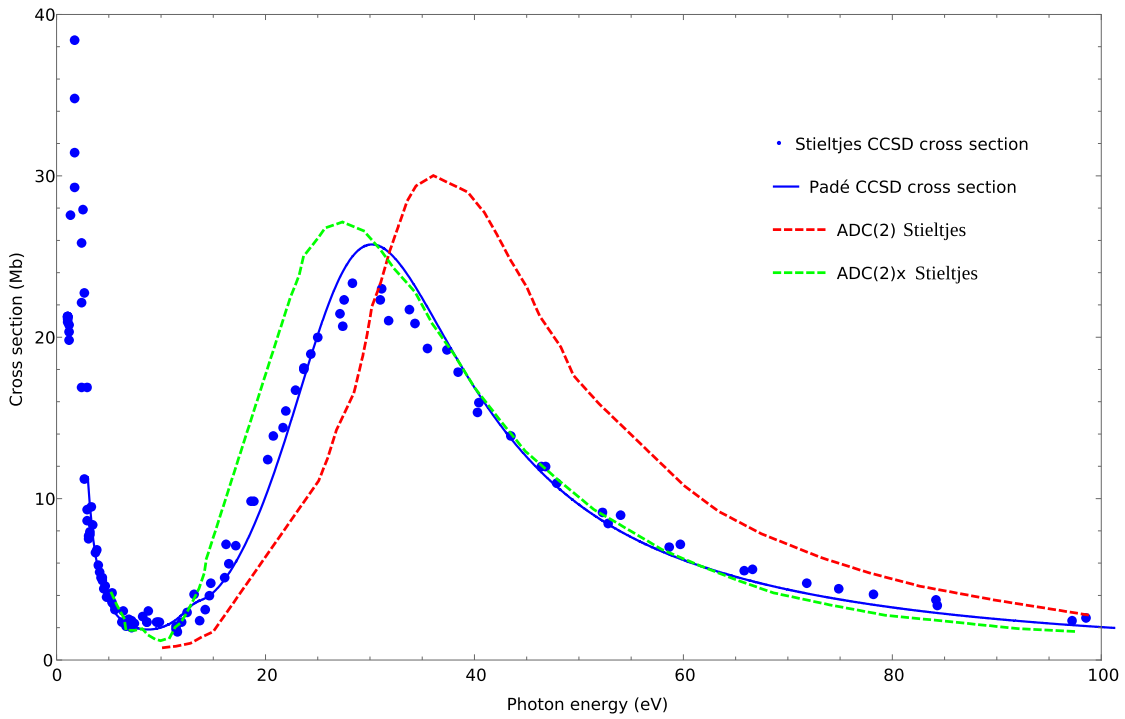
the Padé and Stieltjes imaging procedures. The EOM-CCSD cross sections are compared with the $A D C(2)$ and $A D C(2)-x$ results of Ruberti et al.

geometry of the excited state were calculated at 7.30 and $9.14 \mathrm{eV}$ for the ${ }^{1} B_{1}$ and the ${ }^{1} A_{2}$ states, respectively.

From Fig. 2, one can observe a reasonable agreement between our EOM-CCSD and the ADC(2)-x (Ref. 7) photoionization cross sections of the ${ }^{1} B_{1}$ excited state. In the region above $30 \mathrm{eV}$, the EOM-CCSD and the ADC(2)-x cross sections exhibit the same cross section decay at both geometries considered here. In the region around $20 \mathrm{eV}$, the EOM-CCSD cross section is narrower and slightly shifted, falling in between the $\operatorname{ADC}(2)-x$ and the ADC(2) ones. Analogous behavior is observed for the EOM-CCSD cross section at the lowest-energy geometry of the excited state shown in the bottom panel of Fig. 2. All in all, the EOM-CCSD photoionization cross section agrees better the $\mathrm{ADC}(2)$-x results than with the $\mathrm{ADC}(2)$ ones.

The photoionization cross sections of the ${ }^{1} A_{2}$ excited state, shown in Fig. 3, shows more spectral structures than the ones of the
${ }^{1} B_{1}$ state. In addition, there is a large difference between the results computed at the equilibrium geometry and the ones obtained at the excited state geometry. For example, taking the Padé analytic curves, the maximum cross section $(27 \mathrm{Mb})$ obtained with the ground state equilibrium geometry occurs at $37 \mathrm{eV}$ (24 Mb by the Stieltjes highest point) and at $20 \mathrm{eV}$ with $22 \mathrm{Mb}$ when the calculation is performed at the excited state geometry. This is a clear evidence that the photoionization cross sections from the ${ }^{1} A_{2}$ transient state are much more sensitive to geometry changes than from the ${ }^{1} B_{1}$ excited state.

The cross sections obtained with the analytic Padé procedure are in reasonable agreement with the Stieltjes cross section points. This was also observed in our recent work ${ }^{24}$ where the cross section of inner shell states were obtained within a CC-CVS approximation and the Padé and the Stieltjes cross sections were compared for a number of molecules. Here, the larger difference in the 

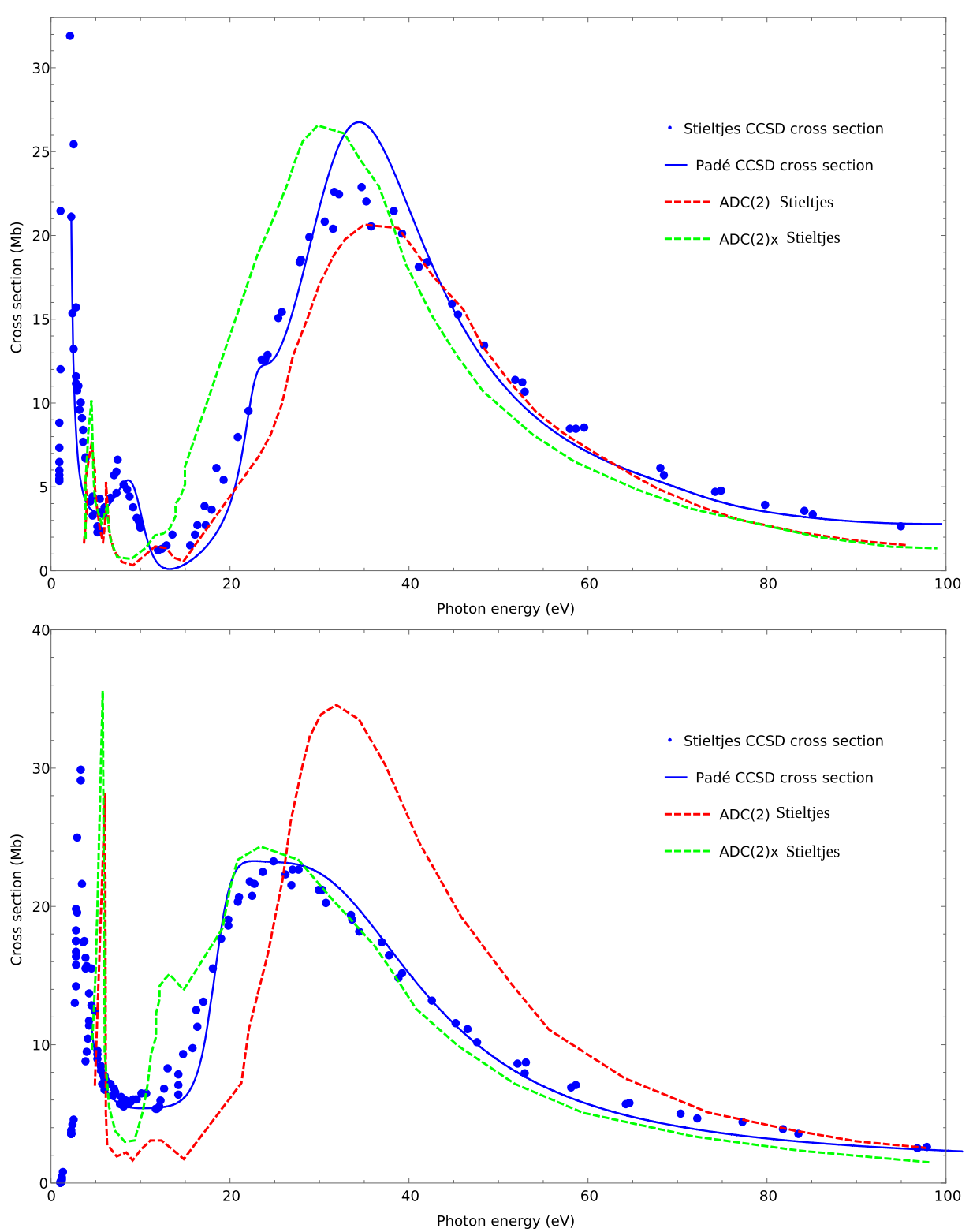

FIG. 3. $\mathrm{H}_{2} \mathrm{O}$. EOM-CCSD photoionization cross sections from the ${ }^{1} A_{2}$ excited state at the ground state equilibrium geometry (top) and at the ${ }^{1} A_{2}$ lowestenergy geometry of the excited-state (bottom) obtained by the Padé and Stieltjes imaging procedures. The EOMCCSD cross sections are compared with the $A D C(2)$ and $A D C(2)-x$ results of Ruberti et al. . $^{\top}$ photoionization cross sections of excited states of water obtained with both procedures was found at the maximum points of the top panels (ground state geometry) of Figs. 2 and 3, where the analytic Padé results are approximately $3 \mathrm{Mb}$ higher.

\section{Carbon monoxide}

The photoionization cross sections of the ${ }^{1} \Sigma^{-}$and ${ }^{1} \Pi$ excited states of carbon monoxide are presented in Figs. 4 and 5, respectively. Similar to the water molecule, we compare our results with the ADC calculations by Ruberti et al. ${ }^{7}$ using the experimental geometries, ${ }^{42}$ i.e., $r_{\mathrm{CO}}=1.128 \AA$ in the ground state and $r_{\mathrm{CO}}=1.391$ and $1.235 \AA$ in the ${ }^{1} \Sigma^{-}$and ${ }^{1} \Pi$ excited state equilibrium geometry, respectively.
The EOM-CCSD vertical excitation energies for the CO were calculated at $10.08 \mathrm{eV}$ and $8.62 \mathrm{eV}$ for the ${ }^{1} \Sigma^{-}$and ${ }^{1} \Pi$ excited states, respectively (see Table I). The results of Ruberti et al. ${ }^{7}$ at the ADC(2)-x and ADC(2) levels are 9.64 and $10.15 \mathrm{eV}$ for the ${ }^{1} \Sigma^{-}$ excited state and 8.32 and $8.85 \mathrm{eV}$ for the ${ }^{1} \Pi$ excited state, respectively. The vertical excitation energies, $9.88 \mathrm{eV}$ for the ${ }^{1} \Sigma^{-}$state and $8.51 \mathrm{eV}$ for the ${ }^{1} \Pi$ state, were obtained numerically by Nielsen, Jørgensen, and Oddershede ${ }^{48}$ using the experimental spectroscopic rovibronic constants obtained from Ref. 42. Our EOM-CCSD adiabatic excitation energies are $8.33 \mathrm{eV}$ for the ${ }^{1} \Sigma^{-}$excited state and $8.24 \mathrm{eV}$ for the ${ }^{1} \Pi$ excited state, while the experimental values are $8.07 \mathrm{eV}$ for both ${ }^{1} \Sigma^{-}$and ${ }^{1} \Pi$ excited states (obtained from Ref. 42 ). Again, our results are in reasonable agreement with the available experimental values. 

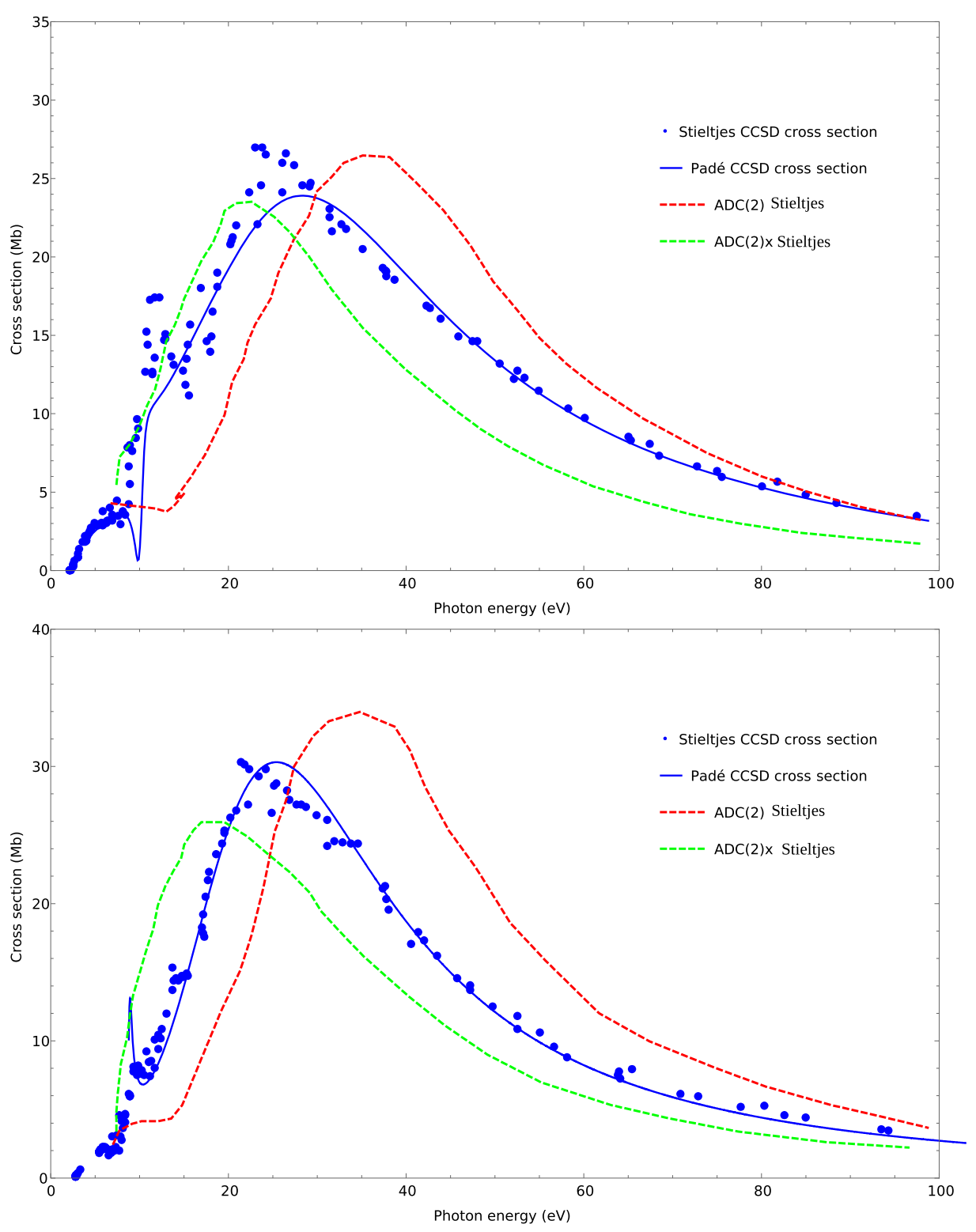

FIG. 4. CO. EOM-CCSD total photoionization cross sections from the ${ }^{1} \Sigma^{-}$ excited state at the ground state equilibrium geometry (top) and at the ${ }^{1} \Sigma^{-}$ excited-state equilibrium geometry (bottom), obtained by the Padé and Stieltjes imaging procedures. The EOM-CCSD cross sections are compared with the $A D C(2)$ and $A D C(2)-x$ results of Ruberti et al.
The cross sections in Fig. 4 for the excited ${ }^{1} \Sigma^{-}$state at the ground state (top panel) and at the excited state geometry (bottom panel) are intermediate between the $\operatorname{ADC}(2)-x$ and $\operatorname{ADC}(2)$ curves, in the whole spectral region, i.e., red shifted relative to the $\mathrm{ADC}(2)$ cross section and blue shifted relative to the $\mathrm{ADC}(2)-\mathrm{x}$ curve. Our analytic Padé cross section calculated at the ground state equilibrium geometry presents a maximum at $28 \mathrm{eV}$ with $24 \mathrm{Mb}$. This maximum is blue shifted by $6 \mathrm{eV}$ relative to the $\mathrm{ADC}(2)-\mathrm{x}$ maximum and red shifted by $10 \mathrm{eV}$ relative to the $\mathrm{ADC}(2)$ maximum. $\mathrm{ADC}(2)-\mathrm{x}$ is known to underestimate the excitation energies due to its unbalanced description of correlation effects in the ground and excited states, which leads to the systematic red shift of the excitation energies. ${ }^{49}$ Conversely, $\operatorname{ADC}(2)$ is known to give a poor description of states with large contributions from double excitations, overestimating Rydberg excitations and ionization; see Ref. 49. CCSD re-establishes the correlation balance and yields excitation energies in between those obtained with the $\mathrm{ADC}(2)-\mathrm{x}$ and the ADC(2) methods.

The results of our calculations for the ${ }^{1} \Pi$ excited state of $\mathrm{CO}$ are shown in Fig. 5 at the ground state equilibrium geometry (top panel) and at the excited state equilibrium geometry (bottom panel). As can be observed from Fig. 5, our EOM-CCSD photoionization cross sections are in better agreement with the $\mathrm{ADC}(2)$-x results than with the $\mathrm{ADC}(2)$ ones $^{7}$ in the whole spectral region, but still in between 

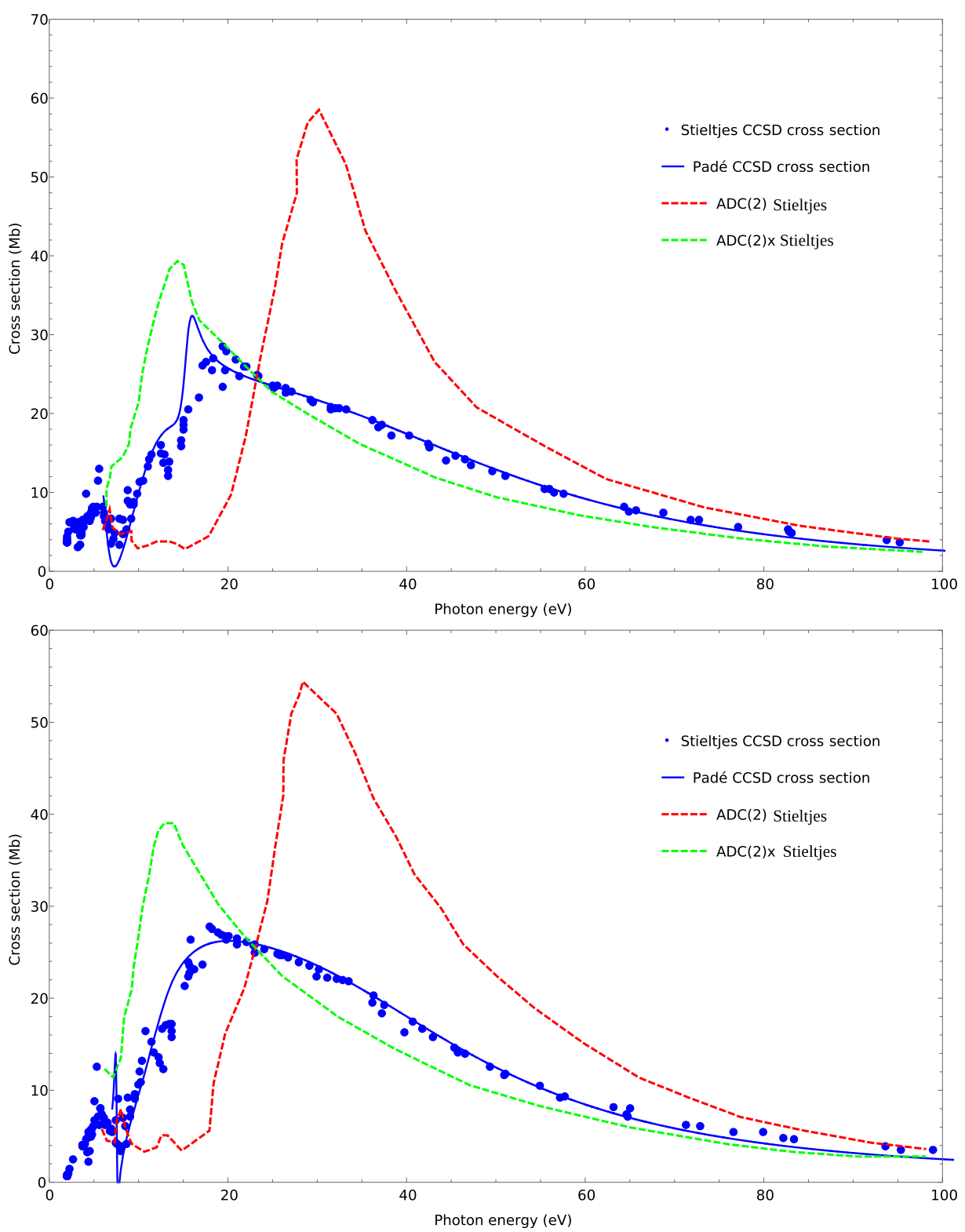

FIG. 5. CO. EOM-CCSD photoionization cross sections from the ${ }^{1} \Pi$ excited state at the ground state equilibrium geometry (top) and at the ${ }^{1} \Pi$ excited state equilibrium geometry (bottom) obtained by the Padé and Stieltjes imaging procedures. The EOM-CCSD cross sections are compared with the $\mathrm{ADC}(2)$ and $A D C(2)-x$ results of Ruberti et al. the maximum positions of the $\operatorname{ADC}(2)-x$ and $\operatorname{ADC}(2)$ curves, due to the more balanced description of correlation effects yielded by the CCSD method.

The photoionization cross section calculated for the transient ${ }^{1} \Pi$ state is not much sensitive to the different geometries, whereas it is possible to see that the EOM-CCSD cross sections as well as the ADC ones, presented in the top and bottom panels of Fig. 5, look very similar to each other.

From Figs. 4 and 5, it is also evident that the analytic Padé photoionization cross sections are in excellent agreement with the Stieltjes cross section points obtained with both geometries and transient states of the CO molecule.

\section{Nitrogen}

The photoionization cross sections of the ${ }^{1} \Sigma_{u}^{-}$and ${ }^{1} \Pi_{g}$ excited states of $\mathrm{N}_{2}$ are presented in Figs. 6 and 7, respectively. Also for this molecule, our results were compared with the $\mathrm{ADC}(2)$ and $\mathrm{ADC}(2)-\mathrm{x}$ ones by Ruberti et al.

The EOM-CCSD vertical excitation energies of $\mathrm{N}_{2}$ are calculated at $10.04 \mathrm{eV}$ and $9.44 \mathrm{eV}$ for the ${ }^{1} \Sigma_{u}^{-}$and ${ }^{1} \Pi_{g}$ excited states, respectively (see Table I). The values obtained at the $\operatorname{ADC}(2)-x$ and $\mathrm{ADC}(2)$ levels ${ }^{7}$ were $10.13 \mathrm{eV}$ and $10.31 \mathrm{eV}$ for the ${ }^{1} \Sigma_{u}^{-}$excited state and $8.98 \mathrm{eV}$ and $9.55 \mathrm{eV}$ for the ${ }^{1} \Pi_{g}$ excited state, respectively. Reference values computed numerically ${ }^{50}$ with experimental rovibronic 

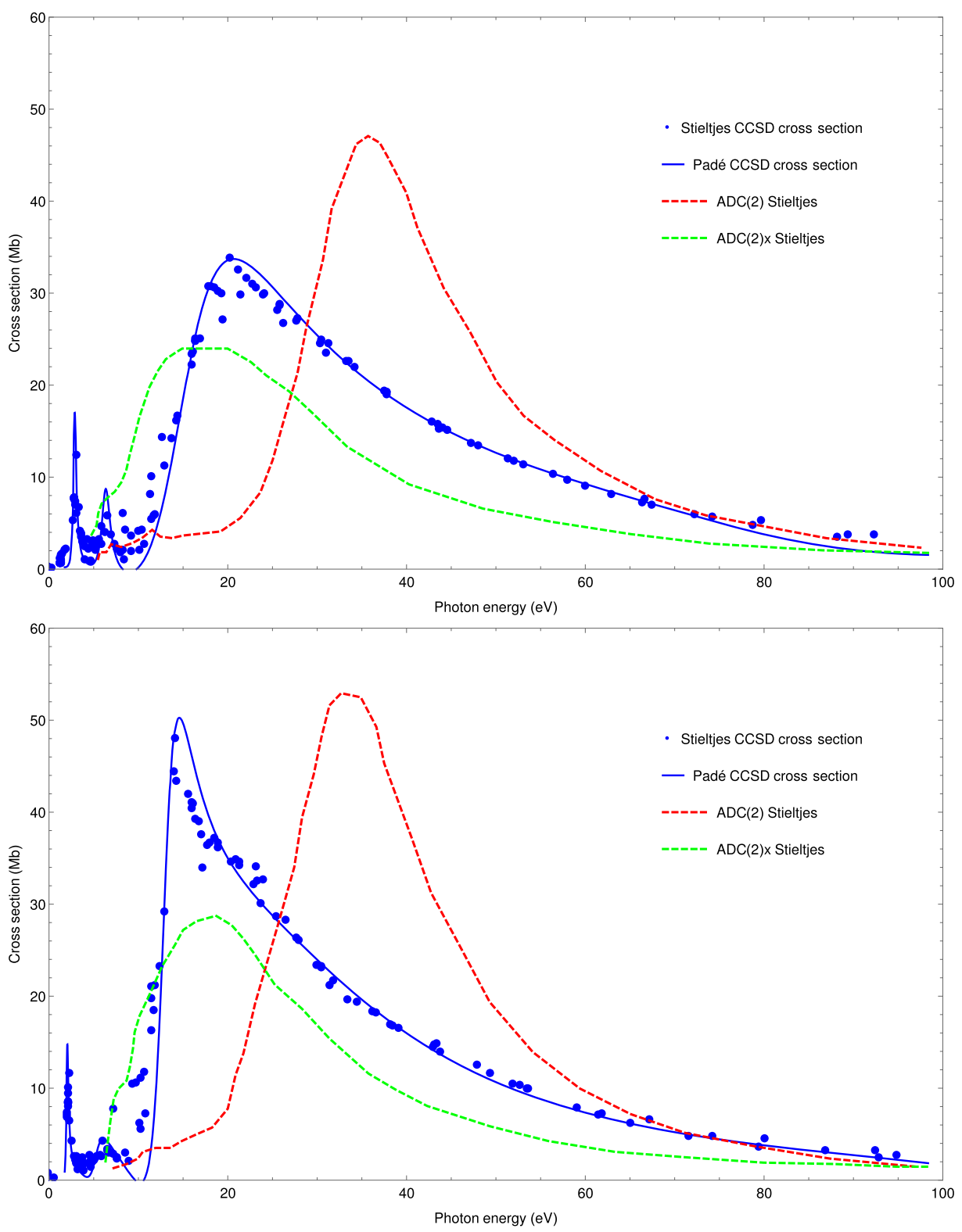

FIG. 6. N2. EOM-CCSD total photoionization cross sections from the ${ }^{1} \Sigma_{u}^{-}$ excited state at the ground state equilibrium geometry (top) and at the ${ }^{1} \Sigma_{u}^{-}$ excited-state equilibrium geometry (bottom), obtained by the Padé and Stieltjes imaging procedures. The EOM-CCSD cross sections are compared with the $A D C(2)$ and $A D C(2)-x$ results of Ruberti et al. constants ${ }^{42}$ were obtained at $9.92 \mathrm{eV}$ and $9.31 \mathrm{eV}$ for the ${ }^{1} \Sigma_{\dot{u}}^{-}$and ${ }^{1} \Pi_{g}$ excited states, respectively. We note that the EOM-CCSD vertical excitation energies are closer to the reference values ${ }^{50}$ than the ones calculated at the $\operatorname{ADC}(2)$ and $\operatorname{ADC}(2)$-x levels. In addition, our EOM-CCSD adiabatic excitation energies are $8.85 \mathrm{eV}$ for the ${ }^{1} \Sigma_{u}^{-}$ excited state and $8.84 \mathrm{eV}$ for the ${ }^{1} \Pi_{g}$ excited state vs the experimental values of 8.59 and $8.45 \mathrm{eV}$ for the ${ }^{1} \Sigma_{u}^{-}$and ${ }^{1} \Pi_{g}$ excited states, ${ }^{42}$ respectively. Once again, our results show reasonable agreement with the experimental data.

In Fig. 6, we present the EOM-CCSD cross sections for the excited ${ }^{1} \Sigma_{u}^{-}$state of $\mathrm{N}_{2}$ at the ground state (top panel) and at the excited state (bottom panel) geometry. The cross section calculated at the ground state equilibrium geometry presents a maximum at $20 \mathrm{eV}$ with $36 \mathrm{Mb}$, which corresponds to a $5 \mathrm{eV}$ blue shift relative to the $\mathrm{ADC}(2)-\mathrm{x}$ maximum and a $15 \mathrm{eV}$ red shift relative to the $\mathrm{ADC}(2)$ maximum. As discussed previously, the better correlation balance in both ground and excited states of the CCSD method $^{49}$ explains the EOM-CCSD cross section curve being in between the ADC ones, similar to what observed in the CO case. In addition, the EOM-CCSD cross section calculated at the ${ }^{1} \Sigma_{u}^{-}$ excited-state geometry (bottom panel) presents a sharp peak with a maximum at $14 \mathrm{eV}$ with $50 \mathrm{Mb}$. This feature is absent in the cross sections obtained at both the $\operatorname{ADC}(2)$ and $\operatorname{ADC}(2)-x$ levels, showing that, for this molecule, the EOM-CCSD cross section is 

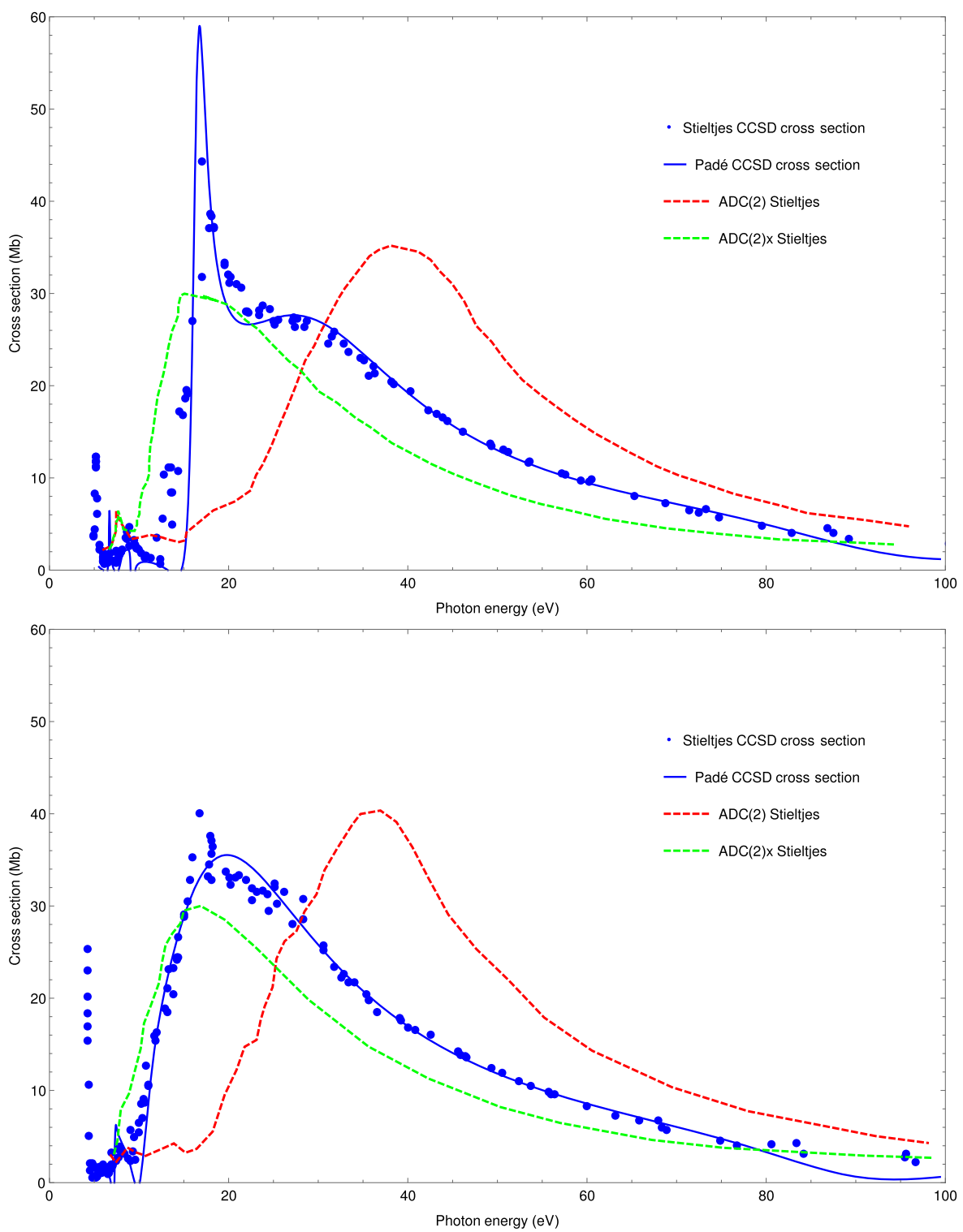

FIG. 7. N2 . EOM-CCSD photoionization cross sections from the ${ }^{1} \Pi_{g}$ excited state at the ground state equilibrium geometry (top) and at the ${ }^{1} \Pi_{g}$ excited state equilibrium geometry (bottom) obtained by the Padé and Stieltjes imaging procedures. The EOM-CCSD cross sections are compared with the $A D C(2)$ and $A D C(2)-x$ results of Ruberti et al.

more sensitive to the molecular geometry variations than the ADC curves.

Our results for the ${ }^{1} \Pi_{g}$ excited state of $\mathrm{N}_{2}$ are presented in Fig. 7, again with those for the ground state equilibrium geometry in the top panel and the ones at the excited state equilibrium geometry in the bottom panel. The EOM-CCSD cross section computed at the ground state geometry shows a sharp peak at $18 \mathrm{eV}$ reaching $60 \mathrm{Mb}$, a value much higher than the maximum in the $\mathrm{ADC}(2)$ curve. This sharp peak is missing in the EOM-CCSD cross section calculated at the ${ }^{1} \Pi_{g}$ excited state geometry (the bottom panel of Fig. 7) using Padé approximants, even though the Stieltjes points appear to still hint at its presence. In this case, the EOMCCSD cross section presents a maximum in an intermediate position between the $\mathrm{ADC}(2)-\mathrm{x}$ and $\mathrm{ADC}(2)$ ones. Roughly, it is possible to say that the EOM-CCSD results shown in Fig. 7 resemble better the characteristics of the $\mathrm{ADC}(2)$-x cross sections than the $\mathrm{ADC}(2)$ ones.

\section{E. Sulfur dioxide}

Sulfur dioxide is a molecule of significant interest in atmospheric chemistry and the astrochemistry related fields of research. ${ }^{51-53}$ Its UV photoabsorption cross section has been studied experimentally and theoretically. ${ }^{53-56}$ The $\mathrm{SO}_{2} \mathrm{UV}$ spectrum exhibits a weak absorption band extending from 3.6 to $5.1 \mathrm{eV}$ dominated by the $\tilde{X}^{1} \mathrm{~A}_{1} \rightarrow{ }^{1} \mathrm{~B}_{1}$ electronic transition, with additional contributions from the $\tilde{X}^{1} \mathrm{~A}_{1} \rightarrow{ }^{1} \mathrm{~A}_{2}$ transition, followed by a strong absorption band extending from 5.4 to $7.3 \mathrm{eV}$ associated with 
the $\tilde{X}^{1} \mathrm{~A}_{1} \rightarrow{ }^{1} \mathrm{~B}_{2}$ electronic transition, which is mainly related to the photodissociation pathway of the $\mathrm{SO}_{2}$ molecule. ${ }^{53}$

At the EOM-CCSD level, we obtained the $\tilde{X}^{1} \mathrm{~A}_{1} \rightarrow{ }^{1} \mathrm{~B}_{1}$ transition at $4.40 \mathrm{eV}$ and with an oscillator strength of 0.0054 , a $\tilde{X}^{1} \mathrm{~A}_{1} \rightarrow 1^{1} \mathrm{~A}_{2}$ transition at $4.87 \mathrm{eV}$, and a $\tilde{X}^{1} \mathrm{~A}_{1} \rightarrow 1^{1} \mathrm{~B}_{2}$ transition at $6.51 \mathrm{eV}$ with oscillator strength 0.079 . The calculated vertical transition energies are in rather good agreement with the experimental data, and we have chosen the first low-lying electronic state $1^{1} \mathrm{~B}_{1}$ and the strongest transition $1^{1} \mathrm{~B}_{2}$ as the target transient states for the excited state photoionization cross section study of the $\mathrm{SO}_{2}$ molecule.

In Fig. 8 , the photoionization cross sections of the $1^{1} \mathrm{~B}_{1}$ (top panel) and $1^{1} \mathrm{~B}_{2}$ (bottom panel) excited states of the $\mathrm{SO}_{2}$ molecule are presented. The correspondent geometries were taken from Ref. 55 as the lowest-energy geometries of the excited states computed at the multireference second order perturbation theory (MRPT2) level with the basis set cc-pVTZ. Our adiabatic electronically excited state energies are 4.02 and $4.61 \mathrm{eV}$ for the $1^{1} \mathrm{~B}_{1}$ and $1^{1} \mathrm{~B}_{2}$ excited states, respectively, while the ones calculated in Ref. 55 with MRPT2 were 4.10 and $5.62 \mathrm{eV}$.

The photoionization cross sections of Fig. 8 show a less pronounced dependence on the molecular geometry than the previous molecules. The results for the transient $1^{1} \mathrm{~B}_{1}$ state (top panel) present a difference of approximately $10 \mathrm{Mb}$ at the maximum of the cross section curve. For the ground state geometry, the maximum value of the cross section, $58 \mathrm{Mb}$, occurs at $27 \mathrm{eV}$, while at the excited state
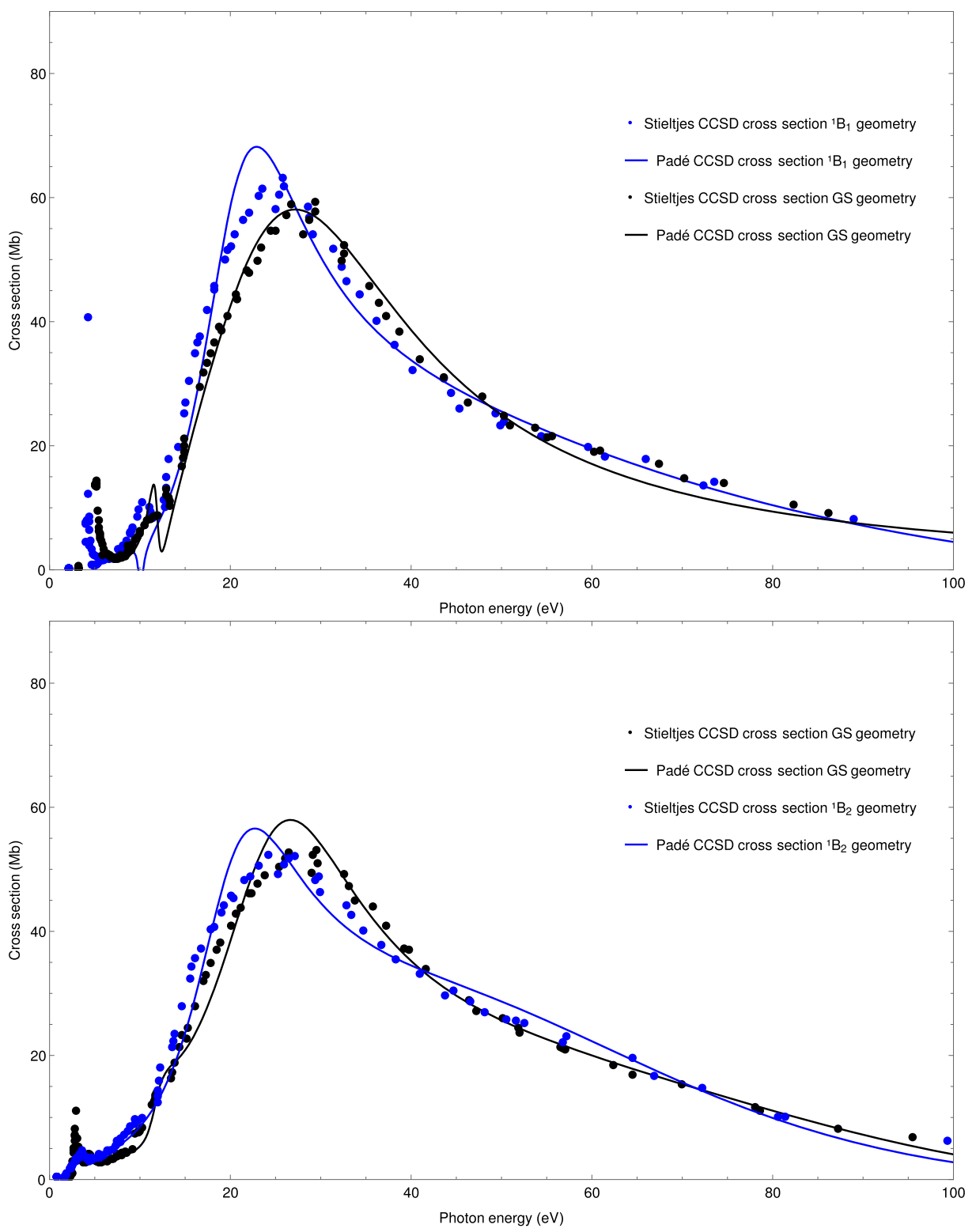

FIG. 8. $\mathrm{SO}_{2}$. EOM-CCSD photoionization cross sections from the ${ }^{1} B_{1}$ (top) and ${ }^{1} B_{2}$ (bottom) excited states, obtained via the Padé and Stieltjes imaging procedures. In the top panel, the results for the ${ }^{1} B_{1}$ excited state at the ground state equilibrium geometry (black) and at the lowest-energy geometry of the excited-state (blue) are shown. In the bottom panel, the cross sections of the ${ }^{1} B_{2}$ excited state at the ground state and relaxed excited state geometries are shown. 
equilibrium geometry the maximum of $68 \mathrm{Mb}$ occurs at $23 \mathrm{eV}$. In the region of higher energy, starting at $40 \mathrm{eV}$, both curves are virtually equivalent. For the transient $1^{1} \mathrm{~B}_{2}$ state (bottom panel), the photoionization cross section calculated at the excited state equilibrium geometry is slightly red shifted (about $2 \mathrm{eV}$ ) relative to the one obtained at ground state geometry. In addition, the maximum of the cross section curves calculated with both geometries occurs at approximately $60 \mathrm{Mb}$ and near $25 \mathrm{eV}$. The results presented in Fig. 8 indicate that the transient photoionization cross section profile of the $\mathrm{SO}_{2}$ molecule is very similar when the molecule is prepared in the $1^{1} \mathrm{~B}_{1}$ or in the $1^{1} \mathrm{~B}_{2}$ excited state.

\section{SUMMARY AND CONCLUSIONS}

We have presented an asymmetric-Lanczos based formulation of EOM-CCSD to compute excitation energies and transition strengths between excited states. The methodology was used to compute the pseudospectra of selected valence excited states in the systems of $\mathrm{He}, \mathrm{H}_{2} \mathrm{O}, \mathrm{CO}, \mathrm{N}_{2}$, and $\mathrm{SO}_{2}$. The pseudospectra were then employed with both the Stieltjes imaging and the Padé analytic continuation procedures to calculate the photoionization cross sections of such transient states. The results were compared with other theoretical results by Ruberti et al. ${ }^{7}$ and with the available experimental data.

For helium, our EOM-CCSD method yields FCI results and the photoionization cross sections calculated for the ${ }^{1} \mathrm{~S}$ and ${ }^{1} \mathrm{P}$ excited states were found in excellent agreement with the experimental data. ${ }^{43,44}$

The CCSD photoionization cross sections of the ${ }^{1} \mathrm{~A}_{2}$ and the ${ }^{1} B_{1}$ transient states of the water, and the ${ }^{1} \Sigma^{-}$and ${ }^{1} \Pi$ excited states of carbon monoxide and of the ${ }^{1} \Sigma_{u}^{-}$and ${ }^{1} \Pi_{g}$ excited states of the nitrogen molecule were found in better agreement with the ones computed at the $\mathrm{ADC}(2)-\mathrm{x} \mathrm{level}^{7}$ than those obtained at the $\mathrm{ADC}(2)$ level. ${ }^{7}$ Generally, when compared to the $\operatorname{ADC}(2)-x$ and $\operatorname{ADC}(2)$ cross sections, the EOM-CCSD results present maxima in between the maxima of the two ADC methods. This behavior can be understood if one considers the unbalanced description of correlation effects in the ground and excited states of the $\operatorname{ADC}(2)-x$ method, leading to a red shift in the $\mathrm{ADC}(2)$-x excitation energies. ${ }^{49} \mathrm{ADC}(2)$, on the other hand, is known to give a poor description of states with large contributions from double excitations overestimating Rydberg excitations and ionization. ${ }^{49}$ As the correlation balance is reestablished by CCSD, the CCSD excitation energies and the photoionization cross sections are found between the $\operatorname{ADC}(2)-x$ and the ADC(2) ones. We also analyzed the dependence of the EOMCCSD cross sections on the geometry of the excited states and demonstrated that it is similar dependence to the one shown by the $\mathrm{ADC}(2)$ - $\mathrm{x}$ cross sections.

The Stieltjes points and the Padé cross section curves were generally in good agreement with each other. In most of the cases, one could even compare the Padé curve to a smooth continuous curve connecting the Stieltjes points. A similar behavior was observed in a previous work, where the photoionization cross sections of inner-shell states of a number of molecules were compared in both methodologies. ${ }^{24}$

We also presented the EOM-CCSD photoionization cross sections for the $1^{1} \mathrm{~B}_{2}$ and the $1^{1} \mathrm{~B}_{1}$ exited states of the sulfur dioxide, a molecule of great interest in atmospheric chemistry and astrochemistry. The $1^{1} \mathrm{~B}_{2}$ exited state is particularly interesting because it is mainly involved in the photodissociation pathway of the $\mathrm{SO}_{2}$ molecule. We observed that, contrary to the case of water and carbon monoxide, the sulfur dioxide cross sections are less dependent on the geometry changes. The photoionization curves showed the maximum cross section values near $25 \mathrm{eV}$ for both the $1^{1} \mathrm{~B}_{2}$ and the $1{ }^{1} \mathrm{~B}_{1}$ excited states.

The approach is presently being extended to the calculation of core-level photoionization cross sections of transient valenceexcited states.

\section{ACKNOWLEDGMENTS}

B.N.C.T., M.A.C.N., and A.B.R. acknowledge Conselho Nacional de Desenvolvimento Científico e Tecnológico (CNPq), Coordenação de Aperfeiçoamento Pessoal de Nível Superior (CAPES) - Finance Code 001, and Fundação de Amparo à Pesquisa do Estado do Rio de Janeiro (FAPERJ) for financial support. S.C. acknowledges support from DTU Chemistry and from the Independent Research Fund Denmark-DFF-Forskningsprojekt2 Grant No. 7014-00258B.

\section{APPENDIX: RIGHT TRANSFORMED EOM-CCSD $\mathrm{A}^{X} R_{i}$ VECTOR}

In the EOM-CC framework, the property Jacobian matrix $\mathbf{A}^{X}$ is different from the CC-LR matrix, as shown in Eq. (6), and the right transformation with the right excitation vector $\left(R_{f}\right)$ reads

$$
\sum_{\mu \nu} \mathbf{A}_{\mu \nu}^{X} R_{v}^{f}=\sum_{\mu \nu} \mathbf{A}_{\mu \nu}^{X, \mathrm{RSP}} R_{v}^{f}+\sum_{\mu \nu}\left\langle\mu\left|\tau_{v} X^{T}\right| \mathrm{HF}\right\rangle\left(1-\delta_{\mu \nu}\right) R_{v}^{f} .
$$

Since the right transformed CC-LR vector $\mathbf{A}_{\mu \nu}^{X, \mathrm{LR}} R_{v}^{f}$ is already available, ${ }^{38}$ we take the second part of Eq. (A1) and treat it as a correction to the CC-LR right transformation. We call this correction term $\sigma_{\mu}$,

$$
\sigma_{\mu}=\sum_{v}\left\langle\mu\left|\tau_{v} X^{T}\right| \mathrm{HF}\right\rangle\left(1-\delta_{\mu v}\right) R_{v}^{f} .
$$

In the CCSD case, $\sigma_{\mu}$ contains contribution for the double excitation manifold $\langle\mu|=\left\langle\begin{array}{c}\tilde{c} d \\ k l\end{array}\right|$, scaled in the biorthonormal basis, and $\tau_{v}=E_{a i}$, where $E_{a i}$ is the singlet one-electron excitation operator. ${ }^{57}$ The CC similarity-transformed operator $X^{T}$ is a component of the electric dipole operator $\hat{X}$ transformed with the cluster operators of single $\left(T_{1}\right)$ and double $\left(T_{2}\right)$ excitation manifold, $X^{T}=e^{-T_{2}-T_{1}} \hat{X} e^{T_{1}+T_{2}}$, and in practice reduces to

$$
X^{T}=X^{T_{1}}+\left[X^{T_{1}}, T_{2}\right]
$$

with the $T_{1}$-transformed operator as

$$
X^{T_{1}}=\sum_{p q} X_{p q}^{T_{1}} E_{p q} .
$$

$X_{p q}^{T_{1}}$ are the $T_{1}$-similarity transformed molecular orbital integrals of the dipole operator ${ }^{57}$ and $T_{2}=\frac{1}{2} \sum_{b e j m} t_{j m}^{b e} E_{b j} E_{e m}$. 
We now rewrite $\sigma_{\mu}$ as

$$
\begin{aligned}
\sigma_{k l}^{c d} & =\sum_{a i}\left\langle\begin{array}{c}
\tilde{c} d \\
k l
\end{array}\left|E_{a i} X^{T}\right| \mathrm{HF}\right\rangle R_{a i}^{f}\left(1-\left\langle\begin{array}{c}
\tilde{c} d \\
k l
\end{array}|i| i\right\rangle\right) \\
& =\sum_{a i}\left\{\left\langle\begin{array}{c}
\tilde{c} d \\
k l
\end{array}\left|E_{a i} X^{T_{1}}\right| \mathrm{HF}\right\rangle+\left\langle\begin{array}{c}
\tilde{c} d \\
k l
\end{array}\left|E_{a i}\left[X^{T_{1}}, T_{2}\right]\right| \mathrm{HF}\right\rangle\right\} R_{a i}^{f} .
\end{aligned}
$$

After some algebraic manipulations, the following expressions are obtained:

$$
\begin{aligned}
\sum_{a i}\left(\begin{array}{l}
\tilde{c} d \\
k l
\end{array}\left|E_{a i} X^{T_{1}}\right| \mathrm{HF}\right) R_{a i}^{f} & =R_{c k}^{f} X_{d l}^{T_{1}}+R_{d l}^{f} X_{c k}^{T_{1}}, \\
\sum_{a i}\left(\begin{array}{c}
\tilde{c} d \\
k l
\end{array}\left|E_{a i}\left[X^{T_{1}}, T_{2}\right]\right| \mathrm{HF}\right\rangle R_{a i}^{f}= & \sum_{e m}\left\{2\left(R_{c k}^{f} X_{m e}^{T_{1}} t_{l m}^{d e}+R_{d l}^{f} X_{m e}^{T_{1}} t_{k m}^{c e}\right)\right. \\
& \left.-\left(R_{c k}^{f} X_{m e}^{T_{1}} t_{l m}^{e d}+R_{d l}^{f} X_{m e}^{T_{1}} t_{k m}^{e c}\right)\right\} .
\end{aligned}
$$

Now, we define an effective amplitude contracted with the oneelectron property integrals

$$
\tilde{t}_{d l}^{X}=\sum_{e m} X_{m e}^{T_{1}}\left(2 t_{l m}^{d e}-t_{l m}^{e d}\right)
$$

which, inserted into Eqs. (A6) and (A5), yields

$$
\begin{aligned}
\sigma_{k l}^{c d} & =R_{c k}^{f}\left(X_{d l}^{T_{1}}+\tilde{t}_{d l}^{X}\right)+R_{d l}^{f}\left(X_{c k}^{T_{1}}+\tilde{t}_{c k}^{X}\right) \\
& =P_{k l}^{c d} R_{c k}^{f}\left(X_{d l}^{T_{1}}+\tilde{t}_{d l}^{X}\right),
\end{aligned}
$$

where we used the permutation operator ${ }^{57} P_{k l}^{c d}$ in the final expression.

The final expression for $\mathbf{A}^{X} R_{f}$ of EOM-CCSD thus reads

$$
\mathbf{A}^{X} R_{f}=\mathbf{A}^{X, \mathrm{RSP}} R_{f}+\sum_{c k, d l} P_{k l}^{c d} R_{c k}^{f}\left(X_{d l}^{T_{1}}+\tilde{t}_{d l}^{X}\right) .
$$

\section{REFERENCES}

${ }^{1}$ M. Hentschel, R. Kienberger, C. Spielmann, G. A. Reider, N. Milosevic, T. Brabec, P. Corkum, U. Heinzmann, M. Drescher, and F. Krausz, Nature 414, 509 (2001). ${ }^{2}$ P. Peng, C. Marceau, and D. M. Villeneuve, Nat. Rev. Phys. 1, 144 (2019).

${ }^{3}$ C. Neidel, J. Klei, C.-H. Yang, A. Rouzée, M. J. J. Vrakking, K. Klünder, M. Miranda, C. L. Arnold, T. Fordell, A. L'Huillier, M. Gisselbrecht, P. Johnsson, M. P. Dinh, E. Suraud, P.-G. Reinhard, V. Despré, M. A. L. Marques, and F. Lépine, Phys. Rev. Lett. 111, 033001 (2013).

${ }^{4}$ P. M. Kraus and H. J. Wörner, Angew. Chem., Int. Ed. 57, 5228 (2018).

${ }^{5}$ H. Wang, M. Chini, S. Chen, C.-H. Zhang, F. He, Y. Cheng, Y. Wu, U. Thumm, and Z. Chang, Phys. Rev. Lett. 105, 143002 (2010).

${ }^{6}$ B. Bernhardt, A. R. Beck, X. Li, E. R. Warrick, M. J. Bell, D. J. Haxton, C. W. McCurdy, D. M. Neumark, and S. R. Leone, Phys. Rev. A 89, 023408 (2014).

${ }^{7}$ M. Ruberti, R. Yun, K. Gokhberg, S. Kopelke, L. S. Cederbaum, F. Tarantelli, and V. Averbukh, J. Chem. Phys. 140, 184107 (2014).

${ }^{8}$ S. P. Neville, V. Averbukh, M. Ruberti, R. Yun, S. Patchkovskii, M. Chergui, A. Stolow, and M. S. Schuurman, J. Chem. Phys. 145, 144307 (2016).

${ }^{9}$ R. J. Squibb, M. Sapunar, A. Ponzi, R. Richter, A. Kivimäki, O. Plekan, P. Finetti, N. Sisourat, V. Zhaunerchyk, T. Marchenko, L. Journel, R. Guillemin, R. Cucini, M. Coreno, C. Grazioli, M. Di Fraia, C. Callegari, K. C. Prince, P. Decleva, M. Simon, J. H. D. Eland, N. Doslic, R. Feifel, and M. N. Piancastelli, Nat. Commun. 9, 63 (2018).

${ }^{10}$ A. Stolow and J. G. Underwood, "Time-resolved photoelectron spectroscopy of nonadiabatic dynamics in polyatomic molecules," in Advances in Chemical Physics (John Wiley \& Sons, Ltd, 2008), Chap. 6, pp. 497-584.

${ }^{11}$ M. Ruberti, P. Decleva, and V. Averbukh, J. Chem. Theory Comput. 14, 4991 (2018).
${ }^{12}$ A. Palacios and F. Martín, Wiley Interdiscip. Rev.: Comput. Mol. Sci. 2019, e1430.

${ }^{13}$ M. Ruberti, R. Yun, K. Gokhberg, S. Kopelke, L. S. Cederbaum, F. Tarantelli, and V. Averbukh, J. Chem. Phys. 139, 144107 (2013).

${ }^{14}$ K. Gokhberg, V. Vysotskiy, L. S. Cederbaum, L. Storchi, F. Tarantelli, and V. Averbukh, J. Chem. Phys. 130, 064104 (2009).

${ }^{15}$ J. Cukras, S. Coriani, P. Decleva, O. Christiansen, and P. Norman, J. Chem. Phys. 139, 094103 (2013).

${ }^{16}$ J. Cukras, P. Decleva, and S. Coriani, J. Chem. Phys. 141, 174315 (2014).

${ }^{17}$ U. Ekström, P. Norman, and V. Carravetta, Phys. Rev. A 73, 022501 (2006).

${ }^{18}$ V. Carravetta, H. Ågren, D. Nordfors, and S. Svensson, Chem. Phys. Lett. 152, 190 (1988).

${ }^{19}$ B. N. C. Tenorio, M. A. C. Nascimento, S. Coriani, and A. B. Rocha, J. Chem. Theory Comput. 12, 4440 (2016).

${ }^{20}$ B. N. C. Tenorio, M. A. C. Nascimento, and A. B. Rocha, J. Chem. Phys. 148, 074104 (2018).

${ }^{21}$ B. N. C. Tenorio, R. R. Oliveira, M. A. C. Nascimento, and A. B. Rocha, J. Chem. Theory Comput. 14, 5324 (2018).

${ }^{22}$ B. N. C. Tenorio, M. A. C. Nascimento, and A. B. Rocha, J. Chem. Phys. 150, 154308 (2019).

${ }^{23}$ D. L. Yeager, M. A. C. Nascimento, and V. McKoy, Phys. Rev. A 11, 1168 (1975).

${ }^{24}$ B. N. C. Tenorio, T. Moitra, M. A. C. Nascimento, A. B. Rocha, and S. Coriani, J. Chem. Phys. 150, 224104 (2019).

${ }^{25}$ F. Pawłowski, J. Olsen, and P. Jørgensen, J. Chem. Phys. 142, 114109 (2015).

${ }^{26}$ J. F. Stanton and R. J. Bartlett, J. Chem. Phys. 98, 7029 (1993).

${ }^{27}$ A. I. Krylov, Annu. Rev. Phys. Chem. 59, 433 (2008).

${ }^{28}$ S. Coriani, F. Pawłowski, J. Olsen, and P. Jørgensen, J. Chem. Phys. 144, 024102 (2016).

${ }^{29}$ R. Faber and S. Coriani, J. Chem. Theory Comput. 15, 520 (2019).

${ }^{30}$ O. Christiansen, P. Jørgensen, and C. Hättig, Int. J. Quantum Chem. 98, 1 (1998).

${ }^{31}$ H. Koch and P. Jørgensen, J. Chem. Phys. 93, 3333 (1990).

${ }^{32}$ S. Coriani, T. Fransson, O. Christiansen, and P. Norman, J. Chem. Theory Comput. 8, 1616 (2012).

${ }^{33}$ S. Coriani, O. Christiansen, T. Fransson, and P. Norman, Phys. Rev. A 85, 022507 (2012).

${ }^{34}$ G. H. Golub and C. F. Van Loan, Matrix Computations, 3rd ed. (Johns Hopkins University Press, Philadelphia, PA, 1996).

${ }^{35}$ P. Langhoff, C. Corcoran, J. Sims, F. Weinhold, and R. Glover, Phys. Rev. A 14, 1042 (1976).

${ }^{36}$ P. Langhoff, Chem. Phys. Lett. 22, 60 (1973).

${ }^{37}$ F. Muller-Plathe and G. H. Diercksen, in Electronic Structure of Atoms, Molecules and Solids, Proceedings of the II Escola Brasileira de Estructure Eletronica, Olinda, Brazil, July 17-22, 1989, edited by S. Canuto, J. D'Albuquerque e Castro, and F. J. Paixao (World Scientific, Olinda, Brazil, 1990), pp. 1-29.

${ }^{38}$ K. Aidas, C. Angeli, K. L. Bak, V. Bakken, R. Bast, L. Boman, O. Christiansen, R. Cimiraglia, S. Coriani, P. Dahle, E. Dalskov, U. Ekström, T. Enevoldsen, J. J. Eriksen, P. Ettenhuber, B. Fernández, L. Ferrighi, H. Fliegl, L. Frediani, K. Hald, A. Halkier, C. Hättig, H. Heiberg, T. Helgaker, A. C. Hennum, H. Hettema, E. Hjertenaes, S. Høst, I.-M. Høyvik, M. F. Iozzi, B. Jansík, H. Jensen, D. Jonsson, P. Jørgensen, J. Kauczor, S. Kirpekar, T. Kjaergaard, W. Klopper, S. Knecht, R. Kobayashi, H. Koch, J. Kongsted, A. Krapp, K. Kristensen, A. Ligabue, O. Lutnæs, J. Melo, K. Mikkelsen, R. Myhre, C. Neiss, C. Nielsen, P. Norman, J. Olsen, J. Olsen, A. Osted, M. Packer, F. Pawlowski, T. Pedersen, P. Provasi, S. Reine, Z. Rinkevicius, T. A. Ruden, K. Ruud, V. V. Rybkin, P. Sałek, C. C. M. Samson, A. S. de Merás, T. Saue, S. Sauer, B. Schimmelpfennig, K. Sneskov, A. H. Steindal, K. O. Sylvester-Hvid, P. R. Taylor, A. M. Teale, E. I. Tellgren, D. P. Tew, A. J. Thorvaldsen, L. Thøgersen, O. Vahtras, M. A. Watson, D. J. D. Wilson, M. Ziolkowski, and H. Ågren, Wiley Interdiscip. Rev.: Comput. Mol. Sci. 4, 269 (2014).

${ }^{39}$ T. H. Dunning, J. Chem. Phys. 90, 1007 (1989).

${ }^{40} \mathrm{~K}$. Kaufmann, W. Baumeister, and M. Jungen, J. Phys. B: At., Mol. Opt. Phys. 22, 2223 (1989). 
${ }^{41}$ J. Páleníková, M. Kraus, P. Neogrády, V. Kellö, and M. Urban, Mol. Phys. 106, $2333(2008)$

${ }^{42}$ K. P. Huber and G. Herzberg, Molecular Spectra and Molecular Structure IV. Constants of Diatomic Molecules (Van Nostrand Reinhold, 1979).

${ }^{43}$ S. Hussain, M. Saleem, and M. A. Baig, Phys. Rev. A 76, 012701 (2007).

${ }^{44}$ M. Gisselbrecht, D. Descamps, C. Lyngå, A. L'Huillier, C.-G. Wahlström, and M. Meyer, Phys. Rev. Lett. 82, 4607 (1999).

${ }^{45}$ V. L. Jacobs, Phys. Rev. A 9, 1938 (1974).

${ }^{46}$ Z.-L. Cai, D. J. Tozer, and J. R. Reimers, J. Chem. Phys. 113, 7084 (2000).

${ }^{47}$ A. Chutjian, R. I. Hall, and S. Trajmar, J. Chem. Phys. 63, 892 (1975).

${ }^{48}$ E. S. Nielsen, P. Jørgensen, and J. Oddershede, J. Chem. Phys. 73, 6238 (1980).

${ }^{49} \mathrm{~B}$. Helmich and C. Hättig, Comput. Theor. Chem. 1040-1041, 35 (2014).

${ }^{50}$ J. Oddershede, N. E. Grūner, and G. H. Diercksen, Chem. Phys. 97, 303 (1985).

${ }^{51}$ J. B. Pollack, O. B. Toon, R. C. Whitten, R. Boese, B. Ragent, M. Tomasko, L. Esposito, L. Travis, and D. Wiedman, J. Geophys. Res.: Space Phys. 85, 8141, https://doi.org/10.1029/ja085ia13p08141 (1980).
${ }^{52}$ J.-L. Bertaux, D. Nevejans, O. Korablev, E. Villard, E. Quémerais, E. Neefs, F. Montmessin, F. Leblanc, J. Dubois, E. Dimarellis, A. Hauchecorne, F. Lefèvre, P. Rannou, J. Chaufray, M. Cabane, G. Cernogora, G. Souchon, F. Semelin, A. Reberac, E. V. Ransbeek, S. Berkenbosch, R. Clairquin, C. Muller, F. Forget, F. Hourdin, O. Talagrand, A. Rodin, A. Fedorova, A. Stepanov, I. Vinogradov, A. Kiselev, Y. Kalinnikov, G. Durry, B. Sandel, A. Stern, and J. Gérard, Planet. Space Sci. 55, 1673 (2007)

${ }^{53}$ D. Blackie, R. Blackwell-Whitehead, G. Stark, J. C. Pickering, P. L. Smith, J. Rufus, and A. P. Thorne, J. Geophys. Res.: Planets 116, 1, https://doi.org/10.1029/2010je003707 (2011).

${ }^{54}$ C. Lévêque, R. Taïeb, and H. Köppel, Chem. Phys. 460, 135 (2015).

${ }^{55} \mathrm{~A}$. Li, B. Suo, Z. Wen, and Y. Wang, Sci. China, Ser. B: Chem. 49, 289 (2006).

${ }^{56}$ P. Nachtigall, J. Hrušák, O. Bludský, and S. Iwata, Chem. Phys. Lett. 303, 441 (1999).

${ }^{57}$ T. Helgaker, P. Jørgensen, and J. Olsen, Molecular Electronic Structure Theory (Wiley, 2004). 